\title{
Adhesive Retained Silicone Auricular Prosthesis in Rehabilitation of Missing Ear
}

\author{
Hema Kanathila ${ }^{1}$, Ashwin Pangi², Bharathi $\mathrm{P}^{3}$ \\ ${ }^{1}$ Department of Prosthodontics, KAHER'S, KLE VK Institute of Dental Sciences, Belagavi, Karnataka, India. \\ ${ }^{2}$ Department of Prosthodontics Aesthetix Dental Clinic Belagavi, Karnataka, India. ${ }^{3}$ Department of Periodontics, \\ Sharavati Dental College, Shivamogga, Karnataka, India.
}

\section{INTRODUCTION}

Auricular anomalies can be categorised into either congenital or acquired defects. Congenital anomalies are because of the flaws in embryogenesis or due to of intrauterine events which affect fetal growth leading to deformations. These deformations can be corrected prosthetically which can be aesthetically pleasing and satisfying for the patients. Auricular prostheses act as a cost-effective and cosmetically acceptable mask for patients for a better quality of social life.

Microtia is a congenital defect where the pinna is under developed. Microtia can be either unilateral or bilateral. Based on the severity of auricular defect, there may be evidence of external meatus formation. In cases of microtia, due to the involvement of the external canal and middle ear, hearing may be affected. ${ }^{1}$ Microtia can be due to hereditary factors or due to some vascular accidents in-utero. It can coexist with cases of Treacher Collins syndrome and Goldenhar's syndrome. ${ }^{2}$ Basically microtia is divided into two main categories. They are lobular type and conchal remnant type microtia. More frequent type is lobular type microtia. ${ }^{1}$

Surgical reconstruction of the defective ear is usually not opted by the patient considering the cost factor, procedures involved and post-operative pain involved. Silicones have been widely used as maxillofacial prosthetic material only for the past few years. The greatest advantage of silicone prosthetic ear is that it allows the defective ear to look as life-like as possible, even though it requires daily care.

Silicone prosthetic ear can be easily adapted to the tissue bed using adhesives which are acceptable and easy for the patients. Adhesives act as good retentive aids and are supplied in various forms as pastes, liquids, emulsions, sprays and doublesided tapes. This paper presents three cases of microtia rehabilitated with silicone auricular prosthesis retained with the adhesive.

Microtia is a congenital defect where the external ear is under developed. A well designed and retentive prosthetic ear can be fabricated to fit over the microtic ear. The role of prosthodontist in fabricating prosthesis, which is functionally and aesthetically acceptable by the patient, is very important.

We present three cases of microtia who have been successfully rehabilitated with adhesive retained silicone auricular prosthesis. Cases of missing ear which had reported to our clinic were restored with silicone auricular prosthesis. Impressions of both sides were made and poured. Wax pattern of the defect side was carved seeing the details of the opposite normal ear. Three piece mould was made. Silicone of appropriate shade was mixed and poured into the mould. Cured prosthesis was removed and extrinsic stains were applied. It was retained on the defect side using silicone adhesive.
Corresponding Author: Dr. Hema Kanathila, Department of Prosthodontics, KAHER's, KLE VK Institute of Dental, Sciences, Belagavi, Karnataka, India. E-mail: hemak_19@yahoo.com

\section{DOI: $10.14260 / j e m d s / 2020 / 785$}

How to Cite This Article:

Kanathila H, Pangi A, Bharathi P, et al. Adhesive retained silicone auricular prosthesis in rehabilitation of missing earcase series. J Evolution Med Dent Sci 2020;9(47):3578-3581, $10.14260 / \mathrm{jemds} / 2020 / 785$

Submission 29-04-2020,

Peer Review 09-10-2020,

Acceptance 15-10-2020,

Published 23-11-2020.

Copyright (C) 2020 Hema Kanathila et al. This is an open access article distributed under Creative Commons Attribution License [Attribution 4.0 International (CC BY 4.0)] 


\section{Clinical Significance}

The use of silicone material in the fabrication of auricular prosthesis has made it possible to restore what has been lost or missing. The success of most non implant retained auricular prostheses depends on the retention. Medical adhesives present as a cost-effective and acceptable retentive aid for patients. The 3 case reports in this article show microtia patients successfully rehabilitated with adhesive retained silicone auricular prosthesis.

\section{PRESENTATION OF CASES}

\section{Case 1}

A 20-year-old male patient reported to our clinic with the complaint of missing right ear. On clinical examination of the patient, it was noted the patient to have a normal ear on left side. He presented to have normal hearing of the same side. On the right side, the patient had a remnant of ear without an auditory canal opening. Hence this patient was diagnosed with microtia of the right side. (Figure 1)

\section{Case 2}

A 15-year-old male patient came to the clinic with congenitally deformed left ear. On evaluation he presented normal hearing with normal formed ear on the right side. On the left side there was a remnant of ear. In this case there was normal auditory canal opening on the left side. (Figure 2)

\section{Case 3}

A 35-year-old male patient came to our clinic presenting a congenitally deformed right ear. On clinical evaluation, he was noted to have a well formed ear on left side. He seemed to have normal hearing with respect to left side ear. A residue of ear was seen on the right side. There was no auditory canal opening on right side. (Figure 3)

\section{FABRICATION OF AURICULAR PROSTHESIS}

Ear is a complex structure. Hence, requires thorough knowledge and skill to sculpture out natural looking ear of the defect side. It involves few procedures which are to be done with utmost attention in order to fabricate an aesthetic prosthesis. To make the prosthesis appear normal, the shape and colour of the prosthesis should reciprocate the natural ear.

\section{Four Steps of Fabrication of Auricular Prosthesis \\ - Impression making \\ - Sculpture of wax model \\ - Obtaining the mould \\ - Colouration and fabrication of the prosthesis}

In order to obtain an impression, petroleum jelly was applied over the skin, hair and the surrounding area of the ear in order to prevent the sticking of impression material. The patient's head was tilted so that the patient's ear is as horizontal to the floor as possible. Alginate impression material was used. Impressions of both sides were made, and a wet gauze was put on it and dental plaster was poured on the alginate impression after it was set in order to provide support for the impression with alginate. The positive replica of both the impressions was obtained. The wax pattern of the defect side was sculptured (Figure 4) out seeing the details on the model of the normal ear. During try in, the fit of the pattern on to the tissue bed, positional alignment with the normal ear, the bulge of the ear pattern and the marginal integrity were checked. The wax pattern is sealed on to the model of the defective side.

A three piece mould was prepared. The first pour is the base for the mould to be prepared; the model of the defect side with wax carving is placed. A separating medium was applied. Four to five grooves were carved out, over and on the rear side of the helix of the wax pattern. Second part of the mould is obtained by blocking the undercut below the helix with dental stone. Later the dental stone was allowed to set. Boxing was done with wax for the third pour. Again separating media (soap solution) was applied and then was filled with dental stone. Dewaxing was done to obtain a three-piece mould in which the fabrication of prostheses is done.

As per manufacturer's instructions the medical grade Room Temperature Vulcanization (RTV) silicone (Cosmosil) was taken and appropriate shade was mixed to match with the contralateral normal ear. This shaded silicone was poured into the mould and rayon fibres were added to give the appearance of microvasculature. After bench curing for 24 hours, cured prosthesis was removed, cleansed and the excess silicone was trimmed. Extrinsic stains were coated to imitate the skin colour of normal ear.
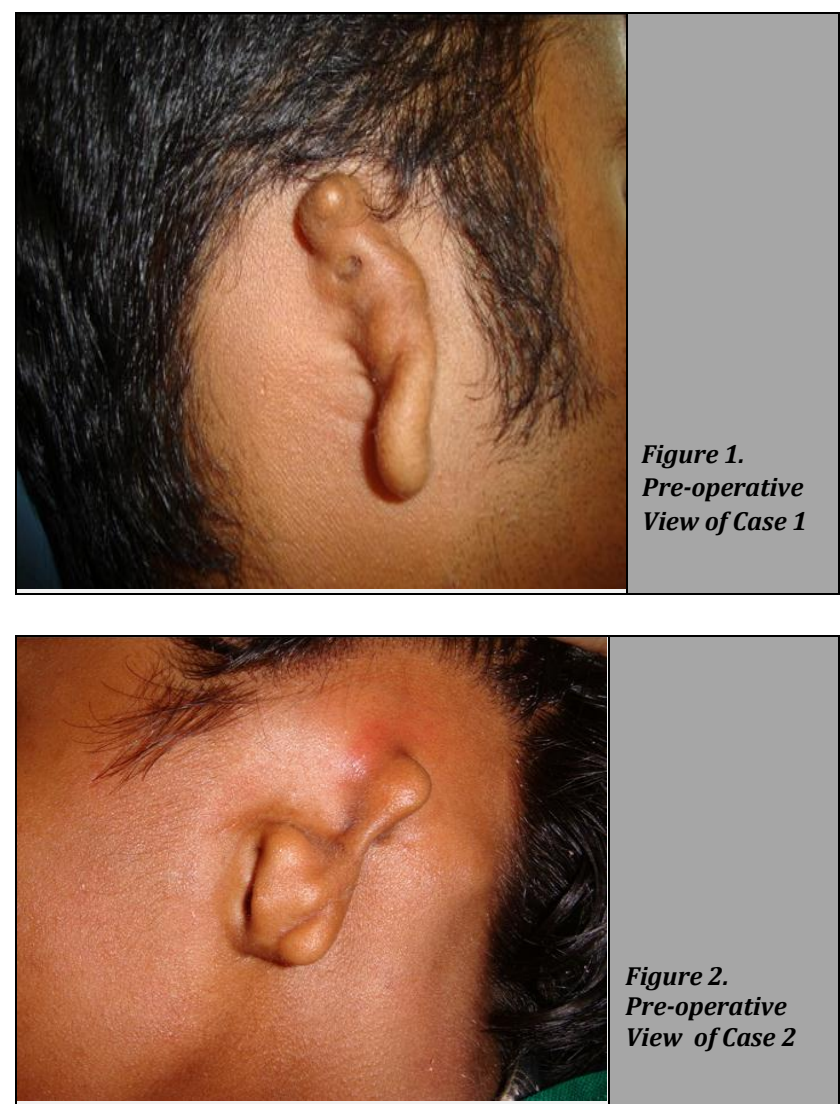

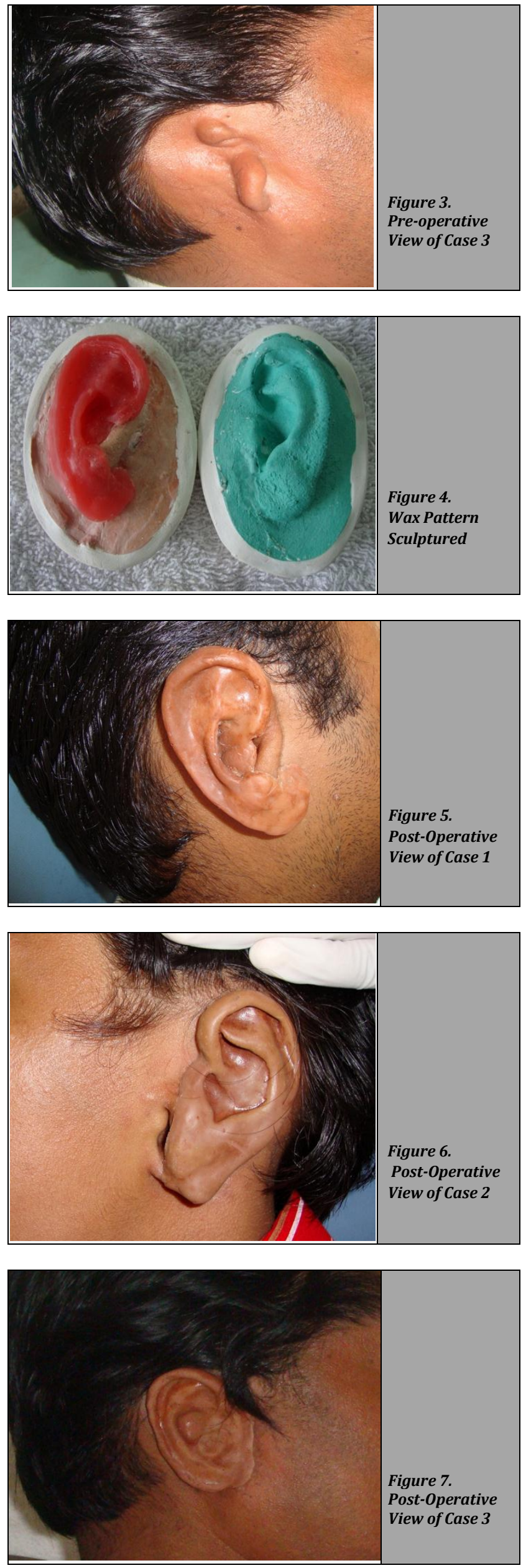

Silicone adhesive was applied as a retentive aid for anchoring the auricular prosthesis onto the tissue bed (Figure 5, 6 and 7). Patients were given instructions on the attachment and removal of the prosthesis and for the daily care of the auricular prosthesis. These patients were instructed to apply the skin adhesive on the tissue surface of the prosthesis and allow it to remain for 2 minutes in order to become further transparent and later to fit the auricular prosthesis over the defect region.

\section{DISCUSSION}

The word "microtia" comes from the latin, where "micro" means little and "otia," means ear, hence "little ear." Apart from congenital and teratogenic causes, acquired causes could be burns, trauma and malignancies which can lead to defective ear. Any defect of the body part makes an individual incapable of leading a normal social life. Hence, such cases require rehabilitation. Replacing the defective part is an art and science and involves time, skill, dedication and knowledge of a prosthodontist.

Silicone rubber for the construction and colouring of facial prostheses was first used by Barnhart. Silicones are considered to be non-toxic product, non-allergenic, easy-toclean, lightweight and compatible with adhesives. Silicones are not too hard, and so produce the elasticity close to the skin as possible. Pigments used with silicone rubber are usually inorganic compounds such as metallic oxides. Tashma used dry earth pigments dispersed in acrylic resin polymer powder for intrinsic staining of a silicone facial prosthesis. ${ }^{3}$

Retention is considered as a key factor in the long term success of the maxillofacial prosthesis. Retention in addition to resistance can be attained by several practices like using adhesives, implants and mechanical aids. ${ }^{4,5}$ GPT 9 defines maxillofacial prosthetic adhesive as "a material used to adhere the external prosthesis to the skin and associated structures around the periphery of an external anatomic defect". In the cases mentioned above, chemical retention is achieved with the use of adhesives. Adhesives are regarded as the most accessible and accepted retentive aid in maxillofacial prosthetic field. Silicone adhesives are a type of roomtemperature vulcanising silicones that are dissolved in a solvent. The mechanism behind the retention provided by it is when the solvent evaporates it results in a tacky adhesive which gives retention. There are certain criteria for the selection of adhesive like biocompatibility, design of the prosthesis, type and quality of patient's skin and bond strength of adhesive to the prosthetic material. ${ }^{6}$ Even though skin adhesives have a disadvantage of getting degraded resulting in decreased strength and bonding ability over a long time and some skin adhesives have shown to cause allergic reactions, the prosthesis which are adhesive retained are more successful. This is because of its easier application technique and low cost compared to implant supported prostheses. ${ }^{7,8}$

\section{CONCLUSIONS}

Rehabilitation of patients with congenital microtia is achieved by restoring the defect and fulfilling the objective of maintaining patient comfort, aesthetics and getting him back 
to his normal social life. The materials for the maxillofacial prosthesis should be chosen by checking their physical and mechanical properties. Hence, attention should be given, both in the choice of materials for the prosthesis and for retention of the prosthesis. The key advantages of silicone auricular prosthesis retained with adhesive is that, the technique is noninvasive, cost effective, good tissue acceptance, aesthetic, comfortable to patient and easy to clean and maintain.

\section{Financial or other competing interests: None.}

Disclosure forms provided by the authors are available with the full text of this article at jemds.com.

\section{REFERENCES}

[1] Lin SJ, Bauer BS, Corcoran J. Microtia. Plastic surgery. Medscape June 29, 2017.

[2] Madhan R, Nayar S. Prosthetic management of patient with treacher collins syndrome. Indian J Dent Res 2006;17(2);78-81.
[3] Barhate AR, Gangadhar SA, Bhandari A, et al. Materials used in maxillofacial prosthesis- a review. Pravara Med Rev 2015;7(1):5-8.

[4] Mardani MA, Arazpour M, Bani MA, et al. Prosthetic rehabilitation of a patient with partial ear amputation using a self-suspension technique. Prosthet Orthot Int 2011;35(4):473-7.

[5] Yeshwante B, Patil SY, Baig N. Retentive aids used in maxillofacial prosthesis. Int J Clin Dent Sci 2014;5(2):1220.

[6] Gurjar R, Kumar MVS, Rao H, et al. Retentive aids in maxillofacial prosthodontics - a review. Int J Contemporary Dent 2011;2(3):84-8.

[7] Padmanabhan TV, Mohamed K, Parameswari D, et al. Prosthetic rehabilitation of orbital and facial defect- a clinical report. J Prosthodont 2012;21(3):200-4.

[8] Somkuwar K, Robin Mathai, Jose P. Ocular prosthesis: patient rehabilitation- a case report. People's J Sci Res 2009;2(2):21-6. 